\title{
Physicians' and Pharmacists' Experience and Expectations of the Roles of Pharmacists: Insights into Hospital Setting in Macau
}

\author{
Ut Mui lo, Hao Hu* and Carolina Oi Lam Ung \\ State Key Laboratory of Quality Research in Chinese Medicine, Institute of Chinese Medical Sciences, University of Macao, \\ Macao, China \\ *For correspondence: Email: haohu@umac.mo
}

\begin{abstract}
Purpose: To investigate physicians' and pharmacists' experience and expectations of the roles of pharmacists in hospital setting in Macau for the development of physician-pharmacist collaborative working relationship (CWR).

Methods: A survey was conducted to address the research questions. The study population included the physicians and pharmacists working in hospitals in Macau. A self-administered survey was designed correspondingly to physicians and pharmacists with same series of questions, which composed of 4 parts: demographics, collaboration status, roles of pharmacist based on experience, and roles of pharmacist based on expectations.

RESULTS: Sixty six out of the 120 physician surveys and 18 out of the 30 pharmacist surveys were returned, giving a response rate of $55.00 \%$ and $60.00 \%$ respectively. $33.33 \%$ of physicians and $77.8 \%$ of pharmacists claimed they collaborated with the other professional at least once a week. The main reason for collaboration was prescription order queries. Both professionals indicated that "medication dispensing" and "identification and prevention of prescription errors" were currently the top responsibilities of pharmacists. It was anticipated by the physicians that pharmacists would remain focused on "medication dispensing" but should put in more effort. Pharmacists, on the other hand, would like to develop their role in direct patient care such as "patient counseling".

Conslusion: There were discrepancies in physicians' and pharmacists' expectations of the roles of pharmacists. The 6 most important responsibilities of pharmacists were determined in consultation with physicians' opinions. Capacity building of pharmacists, communication between the two professionals and administrative co-ordinations were considered important elements in developing CWR.
\end{abstract}

Keywords: Physician-pharmacist relationship, Pharmacist roles, Collaboration, Expectations.

Tropical Journal of Pharmaceutical Research is indexed by Science Citation Index (SciSearch), Scopus, International Pharmaceutical Abstract, Chemical Abstracts, Embase, Index Copernicus, EBSCO, African Index Medicus, JournalSeek, Journal Citation Reports/Science Edition, Directory of Open Access Journals (DOAJ), African Journal Online, Bioline International, Open-J-Gate and Pharmacy Abstracts

\section{INTRODUCTION}

In the light of the innovation in drug development, increasing complexity of treatment regimens and the associated risks of adverse drug events, treating patients with pharmacotherapy safely to reach an optimized clinical outcome has become one of the biggest challenges for physicians. Pharmacists with appropriate competencies can support physicians with reliable drug knowledge and evidence-based medication advice [Error! Reference source not found.,Error! Reference source not found.]. Studies have shown that pharmacists can contribute to the treatment decision-making process in a health care team 
and enhance patient care [Error! Reference source not found.,Error! Reference source not found.]. In a hospital setting where collaboration with allied health care professionals is considered a norm, pharmacists are now held responsible for patient drug safety through direct pharmaceutical care and participation in medication decision-making process, medication management of the hospital and drug knowledge building of the health care team.

The increasingly high expectations for pharmacist are a reflection of the robust evolution in their role specifications. However, the recognition of pharmacists' role in direct patient care remains uncertain and is largely determined by their collaboration with the physicians - the leading role in the multidisciplinary health care team. The Pharmacist-Physician Collaborative Working Relationship (CWR) model was proposed to describe the professional relationship for improving safer medication management and rational pharmacotherapy. Studies have shown that pharmacists in an established CWR were better positioned to utilize their professional knowledge and participate in patient care [Error! Reference source not found.-8]. However, studies have shown that the expanding roles of pharmacists are not widely accepted by physicians, hindering the sustainable development of the profession as well as CWR [Error! Reference source not found.-Error! Reference source not found.]. One of the major reasons is that the contemporary roles of pharmacist have not been clearly established and generally agreed on.

Therefore, this study aims to evaluate the physicians' and pharmacists' experience and expectations of the roles of pharmacists in hospital setting in Macau. It is anticipated that any discrepancies identified will provide a perspective to advance pharmacist's profession and foster the physician-pharmacist CWR to improve the quality of patient care.

\section{METHODS}

\section{Pharmaceutical services of Macau}

Macau is a Special Administrative Region of the People's Republic of China (PRC). Under the policy of "one country, two systems" authorized by PRC, Macau has its own legal system and autonomy. In Macau, the development of the pharmacy profession has been subtle. In 2011, the population of Macau was around 580,000 and the number of registered pharmacists was 309 , giving a pharmacist/1000 population ratio of
0.53 (WHO recommended a ratio of at least 0.5 for optimal health care delivery). The profession was lawfully established in 1990 with the enactment of Decree Law No. 58/90/M (Regulation of pharmacist and pharmacy affairs, Macau) [Error! Reference source not found.]. The law stipulates the pharmacist registration regime and prescribed the fundamental roles for registered pharmacists. Over the years, pharmacists have been striving to establish their professional standing and expand their roles beyond product-oriented duties. In 2010, the government acknowledged the contemporary roles of pharmacists, and consequently, government passed Law No. 6/2010 (Career Regime for Pharmacist). This law enumerated specifically the obligations and different levels of responsibilities in patient care for pharmacists at different seniority in the public health system [Error! Reference source not found.].

\section{Population and settings}

The study population included physicians and pharmacists working in the three hospitals in Macau. Hospital setting was selected because of geographical, organizational, and social proximity of the two professionals, and preestablished role descriptions for pharmacists in a health organization. The numbers of physicians (n1) and pharmacists (n2) in each hospital were: (n1=267, n2=14) in Hospital Centre S. Januario, ( $\mathrm{n} 1=321, \mathrm{n} 2=9)$ in Kiang Wu Hospital, and ( $\mathrm{n} 1=72, \mathrm{n} 2=7)$ in The University Hospital, giving a total of 660 physicians and 33 pharmacists in the hospital setting in Macau. The pharmacist-tophysician ratios in these hospitals were 1:19, $1: 36$ and $1: 10$ respectively.

\section{Data collection}

A survey instrument was used to address the research questions. This survey was approved by the Ethic Committee of University of Macau. According to the small amount of pharmacists in hospitals, a self-administered questionnaire was randomly delivered by mail to 120 physicians and 30 pharmacists working in the 3 hospitals in Macau between January 2013 and April 2013. The questionnaire composed of 4 parts: personal information, current level of collaboration between physicians and pharmacists, physicians'/pharmacists' experience of the pharmacists' role, and physicians' and pharmacists' expectation of the pharmacists' roles. To minimize response bias caused by inappropriate wordings in the survey questions, a pilot test was conducted among 10 hospital physicians and 9 hospital pharmacists, 
thereafter, the survey questions were refined accordingly.

\section{Instrument}

The first part of the questionnaire requested personal information of the participants including age, highest level of education, work location, seniority, etc. The second part consisted of 6 questions which focused on the current physician-pharmacist collaboration status such as frequency and reasons. Frequency of collaboration was divided into 4 categories: "Never", "Rarely", "At least once a week" and "At least once a day" in the survey. The third and the forth part of the survey consisted of 11 questions each, which were designed to investigate the roles of hospital pharmacist perceived by participants based on their experience and expectation, respectively. The respondents were asked to indicate their level of agreement on a 5point scale, in which $1=$ strongly disagree; $2=$ disagree; $3=$ neutral; 4 = agree; and $5=$ strongly agree.

\section{Data analysis}

The data collected were computed and analyzed using the Statistical Package for Social Sciences (SPSS, Version 19) software. Descriptive statistics were calculated for all questions in the survey to establish mean score and standard deviation (SD). Two tailed independent t-tests were used to determine differences in means of variables between physicians and pharmacists. Statistical significance was set at $P$ value $<0.05$.

\section{RESULTS}

\section{Demographics}

The personal information of physician and pharmacist respondents were summarizes as in Table 1. 68 of the 120 physician sampled, completed and returned the questionnaire giving a response rate of $56.7 \%$. However, 2 of the returned questionnaires were excluded due to incompleteness and the successful response rate was reduced to $55.0 \%$. There was equal proportion of both genders among the respondents. The age of physicians ranged from 24 to 72 years with mean age of $34.65 \pm 7.91$. Three physicians had a Ph.D. of degree. The seniority reported was $7.91 \pm 6.91$ years. The median of average working hours per week, workload related to patient care, and number of patients consulted each week were 45 hours, $80 \%$ and 65 patients, respectively.
There were 18 of the 30 pharmacist questionnaires returned, giving a response rate of $60.0 \% .66 .7 \%(n=12)$ of the respondents were female. The age of pharmacists ranged from 24 to 52 years old with mean age and median age of $34.1 \pm 8.85$ and 29.5, respectively. The highest education of pharmacists was Master of Pharmacy and the seniority was $9.96 \pm 8.31$ years. The median of average working hours per week, proportion of working time spent on dispensing, and that on patient care were 40 hours, 20\% and $20 \%$ patients, respectively.

\section{Frequency of collaboration between physicians and pharmacists}

Majority of the physicians $40(60.16 \%)$ said they rarely interact with pharmacists (Table 2). On the other hand, $78 \%$ of pharmacists said they interacted with physicians at least once a week (Table 2). There was a statistically significant difference in frequency of collaboration reported by these two professionals $(P=0.000)$.

\section{Reasons for collaboration}

The higher score represents the more extent of the reason that caused physicians and pharmacists interacted with each other. As shown in Table 3, "Prescription order queries" were the most common reason for collaboration between physicians and pharmacists. For physicians (mean score $=2.62$ ) and pharmacists (mean score $=3.24$ ). The mean scores for each reason for collaboration were similar within each group of respondents, ranging from 2.38 to 2.62 in physician group and 2.33 to 3.24 in the pharmacist group. According to the $P$ values of $t-$ test, there was no statistically significant difference between physicians and pharmacists' reasons for collaboration.

\section{Roles of pharmacist based on participants' experience}

Physicians indicated that, based on experience, pharmacists' involvement in the areas listed in Table 4 was moderate and average (mean scores ranged between 2.38 and 3.45). However, according to the pharmacists, their level of involvement in "Patient education", "Identification and prevention of prescription errors", "Medication dispensing", "Medication record maintenance", "Patient counseling" and "Medication management" was higher than that perceived by the physicians $(p<0.01)$. Both physicians and pharmacists agreed that "Medication dispensing" (mean score of 3.45 and 4.28 respectively) and "Identification and prevention of prescription errors" (mean score of

Trop J Pharm Res, December 2013;12 (6):1079 
3.12 and 4.11 respectively) were the top two priorities for pharmacists. Perceptions of pharmacists and doctors on other roles of pharmacist are shown in Table 4.

Table 1: Personal information of respondents

\begin{tabular}{|c|c|c|}
\hline Demographics & $\begin{array}{l}\text { Physicians, n=66 } \\
\text { n (\%) }\end{array}$ & $\begin{array}{l}\text { Pharmacists, } n=18 \\
\mathrm{n}(\%)\end{array}$ \\
\hline \multicolumn{3}{|l|}{ Age $^{a, b}$} \\
\hline$<30$ & $27(40.91)$ & $8(44.44)$ \\
\hline $31-40$ & $26(39.39)$ & $3(16.67)$ \\
\hline$>40$ & $13(19.7)$ & $3(16.67)$ \\
\hline Others & & $4(22.22)$ \\
\hline \multicolumn{3}{|l|}{ Gender } \\
\hline Male & $33(50)$ & $6(33.3)$ \\
\hline Female & $33(50)$ & $12(66.7)$ \\
\hline \multicolumn{3}{|l|}{ Highest education $^{a}$} \\
\hline Bachelor of Science & $30(45.5)$ & $11(61.1)$ \\
\hline Master of Science & $32(48.5)$ & $6(33.3)$ \\
\hline Doctor of Philosophy & $3(4.5)$ & -- \\
\hline \multicolumn{3}{|l|}{ Seniority } \\
\hline$<5$ & $27(40.9)$ & $5(27.78)$ \\
\hline $6-15$ & $28(42.4)$ & $9(50)$ \\
\hline$>15$ & $11(16.7)$ & $4(22.22)$ \\
\hline \multicolumn{3}{|l|}{ Place of work } \\
\hline Hospital Centre S. Januario & $20(30.3)$ & $7(38.9)$ \\
\hline Kiang Wu Hospital & $37(56.1)$ & $8(44.4)$ \\
\hline The University Hospital & $9(13.6)$ & $3(16.7)$ \\
\hline \multicolumn{3}{|c|}{ Years in Practice (current working place) } \\
\hline$<5$ & $34(51.5)$ & $7(38.89)$ \\
\hline $6-15$ & $26(39.4)$ & $7(38.89)$ \\
\hline$>15$ & $6(9.1)$ & $4(22.22)$ \\
\hline \multicolumn{3}{|l|}{ Current area of Practice } \\
\hline General Physician & $12(18.18)$ & $\mathrm{N} / \mathrm{A}$ \\
\hline Internal Medicine & $9(13.64)$ & \\
\hline Medical department & $2(3.03)$ & \\
\hline Nephrology & $5(7.58)$ & \\
\hline Surgery and Emergency & $22(33.34)$ & \\
\hline Pediatrics & $4(6.06)$ & \\
\hline Others and On training & $12(18.18)$ & \\
\hline
\end{tabular}

a. $\quad$ Four missing values from pharmacists

One missing value from physicians

N/A, not available

Table 2: Frequency of collaboration between physicians and pharmacists

\begin{tabular}{lll}
\hline Frequency of collaboration & Physicians, $\mathbf{n ~ ( \% )}$ & Pharmacists, $\mathbf{n}(\%)$ \\
\hline Never & $4(6.06)$ & $1(5.56)$ \\
Rarely & $40(60.60)$ & $3(16.67)$ \\
At least once a week & $17(25.76)$ & $6(33.33)$ \\
At least once a day & $5(7.58)$ & $8(44.44)$ \\
\hline
\end{tabular}

Table 3: Reasons for collaboration

\begin{tabular}{llllll}
\hline Reasons & Physician & \multicolumn{2}{l}{ Pharmacist $^{*}$ P value $^{*}$} \\
\cline { 2 - 5 } & Mean & SD & Mean & SD & \\
\hline Bio-availability queries $^{b}$ & 2.43 & 1.12 & 2.33 & 1.33 & 0.754 \\
Side-effects queries $^{a, c}$ & 2.55 & 1.15 & 2.44 & 1.20 & 0.894 \\
Drug-alternative queries $^{c}$ & 2.56 & 1.08 & 3.11 & 1.02 & 0.058 \\
Drug-dosage queries $^{c}$ & 2.49 & 1.12 & 2.89 & 1.02 & 0.214 \\
Drug-interaction queries $^{b}$ & 2.38 & 1.13 & 2.44 & 0.78 & 0.798 \\
Prescription order queries $^{a, b}$ & 2.62 & 1.16 & 3.24 & 1.20 & 0.798 \\
\hline
\end{tabular}


$S D$, standard deviation.

a. One missing value from pharmacists

b. One missing value from physicians

c. Two missing values from physicians

${ }^{*} P$ value $<0.05$ is significantly different

Table 4: Roles of pharmacist based on participants' experience

\begin{tabular}{|c|c|c|c|c|c|}
\hline \multirow[t]{2}{*}{ Roles } & \multicolumn{2}{|c|}{ Physicians } & \multicolumn{2}{|c|}{ Pharmacists } & \multirow[t]{2}{*}{$P$ value } \\
\hline & Mean & SD & Mean & SD & \\
\hline Patient education $^{b}$ & 2.85 & 1.18 & 3.83 & 0.92 & $0.001^{*}$ \\
\hline Suggestion of non- prescription medications to patient ${ }^{c}$ & 2.38 & 1.11 & 1.83 & 1.04 & 0.066 \\
\hline Suggestion of prescription medications to patient ${ }^{c}$ & 2.59 & 1.25 & 2.33 & 1.24 & 0.471 \\
\hline Suggestion of prescription medications to physician ${ }^{b}$ & 2.66 & 1.18 & 2.83 & 0.86 & 0.495 \\
\hline Identification and prevention of prescription errors & 3.12 & 1.18 & 4.11 & 0.76 & $0.001^{*}$ \\
\hline Design and monitor of pharmacotherapeutic regimens ${ }^{c}$ & 2.60 & 1.23 & 2.61 & 1.09 & 0.980 \\
\hline Medication dispensing ${ }^{b}$ & 3.45 & 1.29 & 4.28 & 0.89 & $0.002^{*}$ \\
\hline Medication record maintenance ${ }^{a}$ & \multirow{2}{*}{$\begin{array}{l}2.86 \\
2.49\end{array}$} & 1.27 & \multirow{2}{*}{$\begin{array}{l}3.88 \\
2.72\end{array}$} & 0.93 & $0.003^{*}$ \\
\hline$\underset{c}{\text { Development of policies and guidelines for hospital regulations }}$ & & 0.95 & & 0.75 & 0.347 \\
\hline Patient counseling ${ }^{c}$ & \multirow{2}{*}{$\begin{array}{l}2.38 \\
2.97 \\
\end{array}$} & 1.11 & \multirow{2}{*}{$\begin{array}{l}3.39 \\
4.00\end{array}$} & 1.14 & $0.001^{*}$ \\
\hline Medication management & & 1.14 & & 0.91 & $0.001^{*}$ \\
\hline \multicolumn{6}{|c|}{$\begin{array}{l}\text { SD, standard deviation; }{ }^{a} \text { One missing value from pharmacists; }{ }^{D} \text { One missing value from physicians; }{ }^{\circ} \text { Three missing values from } \\
\text { physicians; *'Significantly different }\end{array}$} \\
\hline \multirow[t]{2}{*}{ Roles } & \multicolumn{2}{|c|}{ Physicians } & \multicolumn{2}{|c|}{ Pharmacists } & \multirow[t]{2}{*}{$P$ value } \\
\hline & Mean & SD & Mean & SD & \\
\hline Patient education & 3.38 & 1.25 & 4.50 & 0.62 & 0.000 * \\
\hline Suggestion of non- prescription medications to patient ${ }^{c}$ & 3.06 & 1.13 & 2.94 & 0.94 & 0.685 \\
\hline Suggestion of prescription medications to patient ${ }^{c}$ & 2.91 & 1.19 & 2.83 & 1.25 & 0.356 \\
\hline Suggestion of prescription medications to physician & 3.38 & 1.19 & 3.94 & 0.73 & $0.016^{*}$ \\
\hline Identification and prevention of prescription errors & 3.74 & 1.13 & 4.61 & 0.50 & $0.000^{*}$ \\
\hline Design and monitor of pharmacotherapeutic regimens & 3.39 & 1.16 & 4.11 & 0.68 & $0.002^{*}$ \\
\hline Medication dispensing & 3.80 & 1.14 & 3.83 & 1.29 & 0.923 \\
\hline Medication record maintenance ${ }^{a}$ & 3.59 & 1.14 & 4.39 & 0.77 & $0.005^{*}$ \\
\hline Development of policies and guidelines for hospital regulations ${ }^{b}$ & 3.08 & 1.09 & 3.17 & 0.62 & 0.654 \\
\hline Patient counseling & 3.23 & 1.11 & 4.11 & 0.76 & $0.002^{*}$ \\
\hline Medication management & 3.61 & 1.14 & 4.50 & 0.71 & $0.000^{*}$ \\
\hline
\end{tabular}

SD, standard deviation; ${ }^{a}$ One missing value from pharmacists; ${ }^{\circ}$ One missing value from physicians; ${ }^{\circ}$ Two missing values from physicians; *significantly different.

\section{Roles of pharmacist based on participants' expectation}

Physicians and pharmacists anticipated a higher level of involvement of pharmacists in the areas listed in Table 5. Physicians expected "Medication dispensing" to remain the prime focus of pharmacists, with a mean score of 3.80. On the other hand, pharmacists indicated a different priority. They expected to shift their main focus from "Medication dispensing" (mean score 3.83 as the 6 th priority) to "Identification and prevention of prescription errors" (mean score 4.61), "Patient education" (mean score 4.50) and "Medication Management" (mean score 4.50). Pharmacists also expected greater involvement in all other areas.
Discrepancies in the perception of pharmacists' role based on participants' experience and expectation in each group

Table 6 shows the discrepancies in pharmacists and physicians perception of pharmacists' roles based on experience and expectation. In the

Trop J Pharm Res, December 2013;12 (6):6 
physician group, out of the 11 roles of the pharmacist, 9 of them showed statistically significant differences between perception based on experience and perception based on expectation. Taking into account the higher expectation. Taking into account the higher extent in the future. Likewise in the pharmacist
Table 6: Discrepancies in the perception of pharmacists' roles based on participants' experience and expectation in each group ( $P$ value)

mean scores in Table 5 (expectation) as compared to Table 4 (experience), it was interpreted that physicians would like to see pharmacists perform their duties to a greater extent in the future. Likewise in the pharmacist
roles based on participants' experience and expectation \begin{tabular}{lll}
\hline Roles & Physicians & Pharmacists \\
\hline Patient education & $0.013^{*}$ & $0.016^{*}$ \\
Suggestion of non- prescription medications to patient & $0.001^{*}$ & $0.002^{*}$ \\
Suggestion of prescription medications to patient & 0.144 & 0.236 \\
Suggestion of prescription medications to physician & $0.001^{*}$ & $0.000^{*}$ \\
Identification and prevention of prescription errors & $0.002^{*}$ & $0.026^{*}$ \\
Design and monitor pharmacotherapeutic regimens & $0.000^{*}$ & $0.000^{*}$ \\
Medication dispensing & 0.095 & 0.239 \\
Medication record maintenance & $0.001^{*}$ & 0.169 \\
Development of policies and guidelines for hospital regulations & $0.002^{*}$ & 0.061 \\
Patient counseling & $0.000^{*}$ & $0.032^{*}$ \\
Medication management & $0.002^{*}$ & 0.074 \\
\hline
\end{tabular}

${ }^{*} P$ value $<0.05$ is considered statistically significant difference

group, statistically significant discrepancies between experience and expectation existed in 6 out of 11 roles. The corresponding mean scores in Table 5 were higher than those in Table 4, also suggesting that pharmacists would like to get more involved in the future.

\section{DISCUSSION}

The CWR model describes and differentiates the professional relationship into 5 stages: Stage 0 brief interactions on routine matters; Stage 1 pharmacist start to raise physician's awareness on their services; Stage 2 - physician starts to challenge pharmacist's expertise; Stage 3 scope of collaboration starts to expand and Stage 4 - bilateral communication and mutual trust of both physician and pharmacist have been established with a committed and sustained relationship [Error! Reference source not found.-Error! Reference source not found.,Error! Reference source not found.]. The practice of patient-oriented CWR is determined as Stage 0 in hospitals in Macau. This view is supported by the findings that the majority of physicians never or rarely interacted with pharmacists. Even for physicians who communicated with pharmacists, "Prescription order queries" was the main reason, indicating these two professionals were still working traditionally without collaborative work towards direct patient care. One reason may be that little was known about the contemporary roles of pharmacists or the importance of CWR.

The imbalance in the frequency of collaboration reported by physicians and pharmacists also indicate an issue with health manpower. Our findings show that four-fifth of pharmacists engage in communication with physicians at least once a week. In the light of the small population of pharmacists, this might not indicate a higher level of CWR between the two professionals but an inadequate number of pharmacists in these hospitals. Consequently, pharmacists' functions were limited to their traditional roles of medication dispensing and management, leaving little manpower resources to develop the pharmacist's role in direct patient care in partnership with physicians.

There was a consensus by both professionals about the traditional roles of pharmacists based on what they had experienced. Physicians and pharmacists were certain about pharmacists' main role as the medication dispenser or manager. However, there were statistically significant differences in the level of involvement perceived by the two professionals regarding 6 of the 11 roles on the list. Take "Patient education" as an example, the mean score by physicians and pharmacists were 2.85 and 3.83, respectively. This suggested that while physicians acknowledged that the roles of pharmacists in "Patient education", they considered the need for pharmacist's contribution in this area less relevant. Similarly, the role of pharmacist in "Patient counseling" was considered an important duty by pharmacists, ranking it the top sixth priority, but physicians ranked it as the least important job. The marked discrepancies in the perception of these roles demonstrated that only a few physicians recognized and utilized pharmacists' professional knowledge in provision of direct patient care. 
Physicians' perception about the scope of practice and role priority of pharmacists was very similar based on experience and expectations. Physicians reckoned that "Medication dispensing", "Identification and prevention of prescription errors", "Medication management" should remain the top priorities for pharmacists. Furthermore, physicians expected pharmacists would participate to a great extent in these preestablished roles. This also suggested that a majority of physicians were ignorant about or not confident in pharmacists taking up expanded roles particular in direct patient care. This was in line with experiences in other countries that while many physicians consider pharmacists as drug expert and recognize their traditional roles, only a few of them are comfortable with pharmacists stepping up and providing them with recommendations in the use of prescription medicines or providing direct patient care [Error! Reference source not found.-Error! Reference source not found.].

Different findings were observed in the pharmacist group. Not only did pharmacists expect to perform with greater involvement, they also anticipated re-orientation of their roles shifting towards direct patient care. For pharmacists to have new perceptions about their roles in accordance to international viewpoint was not surprising. Pharmacists in Macau were graduates from medical or pharmacy schools from around the world as there was no local training program for the profession. When graduates who trained in China, U.S., the U.K., Australia, Portugal, Taiwan, etc, returned to Macau, they brought with them up-to-date vision and competencies to carry out professional duties. However, with the profession development lagging behind and lack of appropriate role specification, there was little chance for pharmacists to demonstrate contemporary practice.

While physicians still perceived "Medication dispensing" as pharmacists' prime duty, pharmacists nowadays only participated in this job as a supervisor. There was generally a 2-tier system in any pharmacy practice in Macau which consisted of pharmacists and pharmacy technicians at each tier. Routine medication dispensing was now performed by pharmacy technicians under supervision of pharmacists, which supposedly left pharmacists more time for patient-oriented pharmaceutical care. Although physicians may not consider it appropriate for pharmacists to intervene in patient counseling, marked improvement of patient care was reported in U.S. and Jordan when pharmacists were involved in treatment decision-making process [Error! Reference source not found.,Error! Reference source not found.].

According to the results of pharmacist' experience and expectation on this item, the significant discrepancy showed that pharmacists would like to get involved in more direct patient care. However, physicians on the other hand ranked this area of practice less important based on their expectation. It revealed that although they were aware of the benefits that pharmacists could offer through patient counseling, they were unsure if pharmacists were competent enough to offer reliable medication information and advice to the patients. These again illustrated the issue of lack of trust between the two professionals $[2,3,7]$.

This was of the same importance to physician and pharmacists, now and in the future. This reflected the physicians' recognition of pharmacists as a drug expert. Some studies demonstrated that physicians were comfortable with pharmacists identifying and preventing prescription errors [Error! Reference source not found.,Error! Reference source not found.]. Pharmacists were also determined to prevent medication errors by identifying and solving them in communications with physicians [Error! Reference source not found.]. The Home Medicines Review (HMR) service in Australia is considered a successful model in which an accredited pharmacist is referred by a general practitioner (GP) to visit the patients in their home, review their medicine routine and generate a report for the GP. The GP and patient then agree and act on a medication management plan. HMR which heavily depends on the cooperation between physicians and pharmacists has been shown to successfully address medicine related problems and improve patient's quality of life and health outcomes [Error! Reference source not found.].

Pharmacists' role in clinical setting has been well established in developed countries with the supportive pharmacy law and guideline of pharmacy practice which set forth privileges for pharmacists to perform patient pharmaceutical care [Error! Reference source not found.]. For pharmacists to perform effectively beyond the tradition roles, reorientation of role specifications lawfully or within the health institutes, establishment of code of practice, stipulation of pharmacists' obligation and responsibilities in relation to direct patient care are considered the foremost steps. 
It has been demonstrated in many studies that physicians were receptive to the expanding roles of pharmacists. Pharmacists should therefore be proactive in engaging any form of communication with physicians. Offering them medication advice whenever appropriate through face-to-face communication, research work, information sharing meeting and scientific updates can help to build mutual trust for further collaboration. Both physicians and pharmacists should also learn communication skills which are enormously helpful to resolve the unnecessary conflicts appeared in collaboration [Error! Reference source not found.,Error! Reference source not found.].

Proper continuing professional development for pharmacists is an important element in supporting the development of CWR [Error! Reference source not found.,Error! Reference source not found.,Error! Reference source not found.]. Accreditation by authoritative professional organization of pharmacists' competencies will also help to build pharmacists' credentials which will facilitate trust building by physicians.

Authoritative order is considered crucial for changes to be carried successfully as it does not only come along with instruction, but more importantly all necessary mandatory coordination. Once the administrators recognize the needs and benefits of pharmacists' involvement in direct patient care, they will be in the position to clearly define the pharmacists' roles according to the needs, organize ward rounds by physicians and pharmacists together, grant pharmacists full-access of patient information for purposes of patient counseling and research, adjust human resources to free pharmacists for patient counseling, etc. Suitable initiatives also have to be in place to encourage pharmacists to provide service and generate useful information for the use by physicians.

\section{Limitations of the study and future research}

As with any study, this research has limitations. The response rate of physicians and pharmacists was relatively low and the number of participants from each profession was different, which might have introduced bias. Nonrespondents were neglected which could have been considered and interpreted as the ones with negative attitude towards CWR or who were busy with their work. Despite the pilot test used for refinement of survey questions, translation of the questions might be interpreted differently by participants, which needed further improvement.
Future research should take into account the viewpoints of other stakeholders in CWR in order to obtain more comprehensive information for the development of CWR in Macau. A qualitative study by means of interview of the stakeholders would provide better understanding of their opinions about CWR for further analysis.

\section{CONCLUSION}

This study is significant in two aspects. First of all, this study demonstrated a highly applicable method to identify the direction for development. Secondly, the study provided a roadmap for future development of pharmacist profession based on the expectations of the two professionals in Macau. Findings of this study support the need for role expansion towards direct patient care for pharmacists. While pharmacists' perceptions are the main determinant for reconstructing the profession's scope of practice and role priority, physicians' perception serves the purpose of identifying gaps for executing changes. Despite the discrepancies in physicians' and pharmacists' expectations of the roles of pharmacists, 6 most important responsibilities of pharmacists were determined in consultation with physicians' opinions. In pursuit of an improved CWR, capacity building of pharmacists based on the mutually agreed pharmacists' roles, communication between the two professionals and administrative co-ordinations were considered important elements.

\section{ACKNOWLEDGMENT}

We would like to thanks all the participants in this study. This study was supported by a grant from University of Macau (MYRG044 (Y1-L1)ICMS12-HH).

\section{Conflict of interest}

None of the authors have any conflict of interests to declare

\section{REFERENCES}

1. Wilbur KAB, Beniles A, Hammuda A. Physician perceptions of pharmacist roles in a primary care setting in Qatar. Globalization and Health 2012; 8 . 12.

2. American College of Clinical Pharmacy. The definition of clinical pharmacy. Pharmacotherapy 2008; 28(6): 816-817.

3. SHPA Committee of Specialty Practice in Clinical Pharmacy. SHPA standards of practice for clinical pharmacy. J Pharm Pract Res 2005; 35(2): 122146.

4. World Health Organization. Framework for action on interprofessional education and collaborative 
practice. Geneva, Switzerland 2010a.

5. Zillich AJ, McDonough RP, Carter BL, Doucette WR. Influential characteristics of physician/pharmacist collaborative relationships. Ann Pharmacother 2004; 38: 764-770.

6. McDonough RP. Medication therapy management and collaborative practice: How to make it work. Drug Topics 2011; 115 (12): 52-62.

7. Doucette WR, Nevins J, McDonough RP. Factors affecting collaborative care between pharmacists and physicians. Res Social Adm Pharm 2005; 1 : 567-578.

8. Carter BL, Doucette WR, Franciscus CL, Ardery G, Kluesner KM, Chrischilles EA. Deterioration in BP control following discontinuation of a physician/pharmacist collaborative intervention. Pharmacotherapy 2010; 30(3): 228-235.

9. Awad A, Matowe L, Capps P. Medical doctors perceptions and expectations of the role of hospital pharmacists in Sudan. Pharm World Sci 2007; 29. 557-564.

10. Tahaineh LM, Wazaify M, Albsoul-Younes A, Khader $Y$, Zaidan M. Perceptions, experiences, and expectations of physicians in hospital settings in Jordan regarding the role of the pharmacist. Res Social Adm Pharm 2009; 5: 63-70.

11. Azhar S, Hassali MA, Ibrahim MIM. Doctors' perception and expectations of the role of the pharmacist in Punjab, Pakistan. Trop J Pharm Res 2010; 9(3): 205-222.

12. Hoti K, Sunderland $B$, Hughes J, Parsons R. An evaluation of Australian pharmacist's attitudes on expanding their prescribing role. Pharm World Sci 2010; 32: 610-621.

13. Government, M. S. Decreto-Lei n. ${ }^{\circ} 58 / 90 / M$. In I. O.-G P. Bureau (Ed.), 38/1990. Macau: Macau SAR Government 1990.

14. Macau SAR Government. Decreto-Lei n. ${ }^{\circ}$ 6/2010/M. In I. O.-G. P. Bureau (Ed.), 35/2010. Macau: Macau SAR Government 2010.

15. McDonough RP, Doucette WR. Developing collaborative working relationships between pharmacists and physicians. J Am Pharm Assoc 2001; 41(5): 682 - 692.

16. Australia Government, Department of Human Services. Home Medicines Review (HMR): 2013. Available from:http://www.medicareaustralia.gov.au/provider/ pbs/fifth-agreement/home-medicines-review.jsp [updated 2013 May 27; cited 2013 July 11]

17. Melissa M, Blair JC, Young E. Thrasher K. Pharmacist privileging in a health system: Report of the Qualified Provider Model Ad Hoc Committee. Am J Health Syst Pharm 2007; 64(22): 2373-2381.

18. Sweeney MA. Physician-pharmacist collaboration: $A$ millennial paradigm to reduce medication errors. Am Osteopath Assoc 2002; 102(12): 678-681.

19. American College of Clinical Pharmacy, Shord SS Schwinghammer TL, Badowski $M$, Banderas J, Burton ME, Chapleau CA, Gallagher JC, Matsuura G, Parli SE, Yunker N.. Desired professional development pathways for clinical pharmacists. Pharmacotherapy 2013; 33(4): 34-42. 